\title{
IMPROVED ESTIMATION OF THE COVARIANCE MATRIX OF STOCK RETURNS WITH AN APPLICATION TO PORTFOLIO SELECTION
}

\author{
Olivier Ledoit \\ Michael Wolf
}

00-77

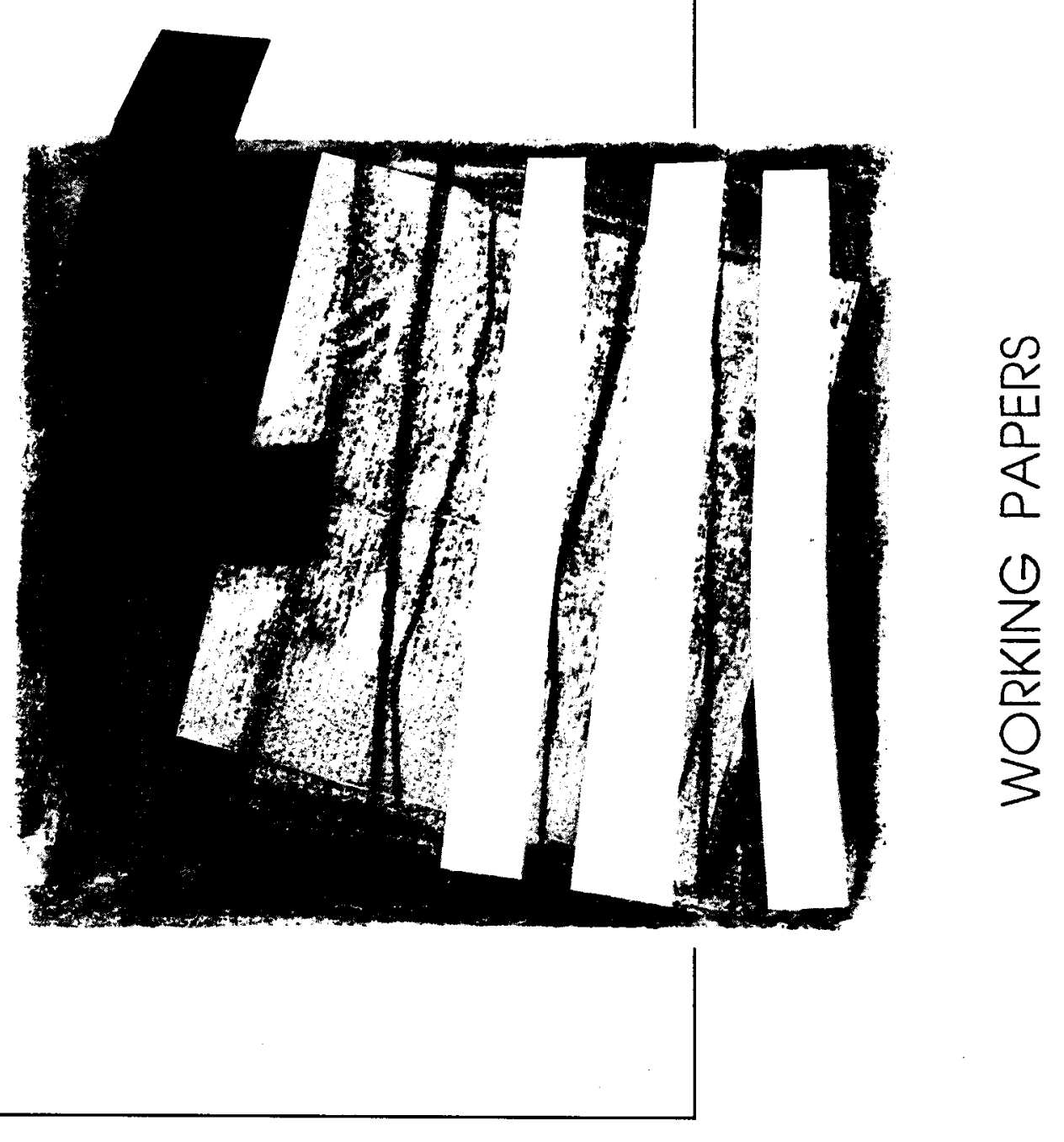

Universidad Carlos III de Madrid 
Working Paper 00-77

Statistics and Econometrics Series 36

November 2000
Departamento de Estadística y Econometría

Universidad Carlos III de Madrid

Calle Madrid, 126

28903 Getafe (Spain)

Fax (34) 91 624-98-49

\title{
IMPROVED ESTIMATION OF THE COVARIANCE MATRIX OF STOCK RETURNS WITH AN APPLICATION TO PORTFOLIO SELECTION
}

Olivier Ledoit and Michael Wolf*

\begin{abstract}
This paper proposes to estimate the covariance matrix of stock returns by an optimally weighted average of two existing estimators. The sample covariance matrix and single-index covariance matrix. This method is generally known as shrinkage, and it is standard in decision theory and in empirical Bayesian statistics. Our shrinkage estimator can be seen as a way to account for extra-market covariance without having to specify an arbitrary multi-factor structure. For NYSE and AMEX stock returns from 1972 to 1995, it can be used to select portfolios with significantly lower out-of-sample variance than a set of existing estimators, including multifactor models.
\end{abstract}

Keywords: Covariance matrix estimation; Factor models; Portfolio selection; Shrinkage method.

*Ledoit, Anderson Graduate School of Management, UCLA, USA; Wolf, Departamento de Estadística y Econometría, Universidad Carlos III de Madrid, C/ Madrid 12628903 Getafe, Madrid, Spain, e-mail: mwolf@est-econ.uc3m.es, Phone: 34-91-6249893. We wish to thank Andrew Lo, Hohn Heaton, Bin Zhou, Timothy Crack, Bruce Lehmann, Richard Michaud, Richard Roll, Pedro Santa-Clara and Jay Shanken for their feedback. Also, the paper has benefited from seminar participants at MIT, the NBER, UCLA, Washington University in Saint Louis, Yale, Chicago, Wharton and UBC. All remaining errors are our own. Research of the second author partly funded by the Spanish "Dirección General de Enseñanza Superior" (DGES), reference number PB98-0025. 


\section{Introduction}

The objective of this paper is to estimate the covariance matrix of stock returns. This is a fundamental question in empirical Finance with implications for portfolio selection and for tests of asset pricing models such as the CAPM.

The traditional estimator - the sample covariance matrix - is seldom used because it imposes too little structure. When the number of stocks $N$ is of the same order of magnitude as the number of historical returns per stock $T$, the total number of parameters to estimate is of the same order as the total size of the data set, which is clearly problematic. When $N$ is larger than $T$, the sample covariance matrix is always singular, even if the true covariance matrix is known to be non-singular. ${ }^{1}$

These severe problems may come as a surprise, since the sample covariance matrix has appealing properties, such as being maximum likelihood under normality. But this is to forget what maximum likelihood means. It means the most likely parameter values given the data. In other words: let the data speak (and only the data). This is a sound principle, provided that there is enough data to trust the data. Indeed, maximum likelihood is justified asymptotically as the number of observations per variable goes to infinity. It is a general drawback of maximum likelihood that it can perform poorly in small sample. For the covariance matrix, small sample problems occur unless $T$ is at least one order of magnitude larger than $N$.

The cure is to impose some structure on the estimator. Ideally, the particular form of the structure should be dictated by the problem at hand. In the case of stock returns, a low-dimensional factor structure seems natural. But this leaves two very important questions: How much structure should we impose? And what factors should we use?

To address these questions properly, we have to be more specific about how we impose a low-dimensional factor structure. One possible way is to specify a $K$-factor model with uncorrelated residuals. Then $K$ controls how much structure we impose: the fewer the factors, the stronger the structure. The advantages of this approach are that it is quite familiar to the Finance profession, and that the factors sometimes have economic interpretation. The disadvantages are that there is no consensus on the identity of the factors - except for the first one, which represents a market index -, and that there is no consensus on the number of factors $K$ either (Connor and Korajczyk, 1992). In other words, choosing between factor models is very ad hoc. It does not mean that none of them works well, it means that we do not know which one works well a priori. For example, if we are interested in selecting portfolios with low out-of-sample variance, in any given data set there may exist a factor model that performs well, but it may be a different one for every data set, and there is no way of telling which one works well without looking out-of-sample, which is cheating. The art of choosing a factor model adapted to a given data set without seeing its out-of-sample fit is just that: an art.

\footnotetext{
${ }^{1}$ In typical applications, there can be over a thousand stocks to choose from, but rarely more than ten years of monthly data, i.e. $N=1,000$ and $T=120$.
} 
This is why, in this paper, we study another way of imposing factor structure. It is to take a weighted average of the sample covariance matrix with Sharpe's (1963) single-index model estimator. The weight $\alpha$ (between zero and one) assigned to the single-index model controls how much structure we impose: the heavier the weight, the stronger the structure. This is a well-known technique in Statistics called shrinkage dating back to Stein (1956): $\alpha$ is called the shrinkage intensity, and the single-index model is our choice of shrinkage target. The advantages are that there is strong consensus on the nature of the single factor (a market index), and that there is a way of estimating the optimal shrinkage consistently. The estimation of $\alpha$ is the technically challenging part of this paper. It provides a rigorous answer to the question of how much structure we should impose. On any given data set, there will be a different optimal shrinkage intensity, and our estimation technique will find it without having to look out-ofsample. This takes the ad-hockery out of the task of imposing structure on the covariance matrix of stock returns. It replaces the art of factor selection by a fully automatic procedure.

At this point, it is worth mentioning that the paper is solely concerned with the structure of risk in the stock market, not with the structure of expected returns. Multi-factor models of the covariance matrix can still be very useful if economic arguments tie them up to the cross-section of expected returns, as in the Arbitrage Pricing Theory of Ross (1976). Any discussion of the relationship between risk factors and expected returns is outside the scope of the paper. There should be no ambiguity over whether we define "factors" in terms of the mean vector or of the covariance matrix of stock returns: it is always the latter.

Muirhead (1987) reviews the large literature on shrinkage estimators of the covariance matrix in finite-sample statistical decision theory. All these estimators suffer from at least two severe drawbacks, either of which is enough to make them ill-suited to stock returns: (i) they break down when $N>T$; (ii) they do not exploit the a priori knowledge that stock returns tend to be positively correlated to one another. Frost and Savarino (1986) show that the solution to the second problem is to use a shrinkage target that incorporates a market factor, but they ignore without justification the correlation between estimation error on the shrinkage target and on the covariance matrix, and they are still exposed to the first problem. A main contribution of our paper to the literature is to address the first problem through the definition the optimal shrinkage intensity by minimizing a loss function that does not involve the inverse of the covariance matrix. Moreover, the technique is so general that it is applicable to other shrinkage targets as well.

A noteworthy innovation is that the optimal shrinkage intensity depends on the correlation between estimation error on the sample covariance matrix and on the shrinkage target. Intuitively, if the two of them are positively (negatively) correlated, then the benefit of combining the information that they contain is smaller (larger). The introduction of this correlation term resolves a deep logical inconsistency in earlier empirical Bayesian literature, where the prior is estimated from sample data, yet at the same time is assumed to be independent from sample data.

We test the performance of our shrinkage estimator on stock returns data for portfolio 
selection. Using NYSE and AMEX stocks from 1972 to 1995, we find that our estimator yields portfolios with significantly lower out-of-sample variance than a set of well-established competitors, including multi-factor models.

The remainder of the paper is organized as follows. Section 2 presents our shrinkage estimator of the covariance matrix. Section 3 presents empirical evidence on its out-of-sample performance for portfolio selection. Finally, Section 4 concludes.

\section{Shrinkage Estimator of the Covariance Matrix}

This section presents the covariance matrix estimator that we recommend for stock returns.

\subsection{Statistical Model}

Let $\mathrm{X}$ denote an $N \times T$ matrix of $T$ observations on a system of $N$ random variables representing $T$ returns on a universe of $N$ stocks.

Assumption 1 Stock returns are independent and identically distributed (iid) through time.

Even though actual stock returns do not verify Assumption 1, it is an acceptable first-cut approximation. This means that we abstract from lead-lag effects (Lo and MacKinlay, 1990), nonsynchronous trading (Shanken, 1987), and autoregressive conditional heteroskedasticity (Bollerslev, Engle and Woooldridge, 1988). Note that most of the current estimators for the covariance matrix of stock returns also use this assumption. Future research will be devoted to relaxing it. It is, however, not clear that by introducing extra degrees of freedom in the estimation process to account for dependence and conditional heteroskedasticity one will be able to improve out-of-sample performance.

Assumption 2 The number of stocks $N$ is fixed and finite, while the number of observations $T$ goes to infinity.

Assumption 3 Stock returns have finite fourth moment:

$$
\forall i, j, k, l=1, \ldots, n \quad \forall t=1, \ldots, T \quad \mathrm{E}\left[\left|\mathrm{x}_{\mathrm{it}} \mathrm{x}_{\mathrm{jt}} \mathrm{x}_{\mathrm{kt}} \mathrm{x}_{\mathrm{lt}}\right|\right]<\infty
$$

This is so that we can apply the Central Limit Theorem to sample variances and covariances. Note that stock returns are not assumed to be normally distributed in this paper. 


\subsection{Sample Covariance Matrix}

The sample mean vector $\mathbf{m}$ and the sample covariance matrix $\mathbf{S}$ are defined by:

$$
\begin{aligned}
\mathbf{m} & =\frac{1}{T} \mathbf{X} \mathbf{1} \\
\mathbf{S} & =\frac{1}{T} \mathbf{X}\left(\mathbf{I}-\frac{1}{T} \mathbf{1 1 ^ { \prime }}\right) \mathbf{X}^{\prime}
\end{aligned}
$$

where 1 denotes a conformable vector of ones and $I$ a conformable identity matrix. ${ }^{2}$ Equation (2) shows why the sample covariance matrix is not invertible when $N \geq T$ : the rank of $\mathrm{S}$ is at most equal to the rank of the matrix $\mathrm{I}-11^{\prime} / T$, which is $T-1$. Therefore when the dimension $N$ exceeds $T-1$, the sample covariance matrix is rank-deficient. Intuitively, the data do not contain enough information to estimate the unrestricted covariance matrix.

\subsection{Single-Index Covariance Matrix Estimator}

Sharpe's (1963) single-index model assumes that stock returns are generated by:

$$
x_{i t}=\alpha_{i}+\beta_{i} x_{0 t}+\varepsilon_{i t}
$$

where residuals $\varepsilon_{i t}$ are uncorrelated to market returns $x_{0 t}$ and to one another. Also, within stocks the variance is constant, that is, $\operatorname{Var}\left(\varepsilon_{i t}\right)=\delta_{i i}$. The covariance matrix implied by this model is:

$$
\Phi=\sigma_{00}^{2} \boldsymbol{\beta} \boldsymbol{\beta}^{\prime}+\Delta
$$

where $\sigma_{00}^{2}$ is the variance of market returns, $\boldsymbol{\beta}$ is the vector of slopes, and $\boldsymbol{\Delta}$ is the diagonal matrix containing residual variances $\delta_{i i}$. Call $\phi_{i j}$ the $(i, j)$-th entry of $\mathbf{\Phi}$.

This model can be estimated by running a regression of stock $i$ 's returns on the market. Call $b_{i}$ the slope estimate and $d_{i i}$ the residual variance estimate. Then the single-index model yields the following estimator for the covariance matrix of stock returns:

$$
\mathbf{F}=s_{00}^{2} \mathbf{b b}^{\prime}+\mathbf{D}
$$

where $s_{00}^{2}$ is the sample variance of market returns, $\mathbf{b}$ is the vector of slope estimates, and $\mathbf{D}$ is the diagonal matrix containing residual variance estimates $d_{i i}$. Call $f_{i j}$ the $(i, j)$-th entry of $\mathbf{F}$. We need to make two technical assumptions.

\section{Assumption $4 \Phi \neq \Sigma$}

Assumption 5 The market portfolio has positive variance, that is, $\sigma_{00}^{2}>0$.

The exact composition of the market portfolio is not as critical here as it is for the CAPM (Roll, 1977). All we need is for $x_{0 t}$ to explain a significant part of the variance of most stocks,

\footnotetext{
${ }^{2}$ Vectors (matrices) are denoted in lower (upper) case boldface.
} 
and any broad-based market index would do. As a matter of fact, equal-weighted indices are better at explaining stock market variance than value-weighted indices, yet another departure from CAPM intuition. The assumption that residuals are uncorrelated to one another should theoretically preclude that the portfolio which makes up the market contain any of the $N$ stocks in the sample. However, as long as the size of the portfolio is large, such a violation will have a very small effect and is typically ignored in applications.

\subsection{General Form of the Shrinkage Estimator}

At one extreme, the single-index covariance matrix comes from a one-factor model, while at the other extreme, the sample covariance matrix can be interpreted as an $N$-factor model (each stock being a factor, there are no residuals). The intuition of the profession has always been that the best model lies somewhere between these two extremes. For example, Rosenberg (1974) stresses the importance of extra-market covariance, while Jobson and Korkie (1980) document the poor performance of the sample covariance matrix. Until now, this intuition has been expressed mostly through $K$-factor models with $1<K<N$.

If we abstract ourselves from the Finance context and look at the broader picture from a statistician's point of view, we see another way of capturing the same intuition. The key is to recognize that the single-index model covariance matrix has a lot of bias coming from a stringent and misspecified structural assumption, but little in the way of estimation error, and that the opposite is true of the sample covariance matrix: it is unbiased (asymptotically) but has a lot of estimation error. A fundamental principle of statistical decision theory is that there exists an interior optimum in the trade-off between bias and estimation error. Since Stein's (1956) seminal work, we know that one way of attaining this optimal trade-off is simply to take a properly weighted average of the biased and unbiased estimators. This is called shrinking the unbiased estimator full of estimation error towards a fixed target represented by the biased estimator. For example, Stein (1956) showed that shrinking sample means towards a constant can, under certain circumstances, improve accuracy. Efron and Morris (1977) provide a general introduction to shrinkage, and Jorion (1986) shows its importance in the context of portfolio selection.

Here this well-established statistical method suggests taking a weighted average of the single-index model covariance matrix and the sample covariance matrix. This is an alternative way of capturing the intuition that led to the development of multi-factor models. The main advantage of this alternative is that it does not require knowledge of the number and nature of factors (beyond the obvious market factor).

\subsection{Formula for the Optimal Shrinkage Intensity}

The obvious problem in the application of our method is the selection of the shrinkage intensity. This section discusses the optimal shrinkage intensity and its consistent estimation from the data. Since $N$ is fixed and $T$ goes to infinity, $\mathbf{S}$ is consistent but $\mathbf{F}$ is not, therefore the optimal 
shrinkage intensity vanishes asymptotically. As will be shown below, it is of the (expected) order $O(1 / T)$. To simplify the estimation, we therefore will focus on shrinkage intensities of the form $\alpha=$ constant/T. The goal then becomes to first find the optimal constant and to then estimate it consistently in order to arrive at a feasible shrinkage estimator.

We have to choose the objective according to which the shrinkage intensity is "optimal." All existing shrinkage estimators from finite-sample statistical decision theory and also Frost and Savarino's (1986) break down when $N \geq T$ because their loss functions involve the inverse of the covariance matrix. Instead, we propose a loss that does not depend on this inverse. The loss function is extremely intuitive: it is a quadratic measure of distance between the true and the estimated covariance matrices based on the Frobenius norm.

Definition 1 The Frobenius norm of the $N \times N$ symmetric matrix $\mathbf{Z}$ with entries $\left(z_{i j}\right)_{i, j=1, \ldots, N}$ and eigenvalues $\left(\lambda_{i}\right)_{i=1, \ldots, N}$ is defined by:

$$
\|\mathbf{Z}\|^{2}=\operatorname{Trace}\left(\mathbf{Z}^{2}\right)=\sum_{i=1}^{N} \sum_{j=1}^{N} z_{i j}^{2}=\sum_{i=1}^{N} \lambda_{i}^{2}
$$

By considering the Frobenius norm of the difference between the shrinkage estimator and the true covariance matrix, we arrive at the following quadratic loss function:

$$
\mathrm{L}(\alpha)=\|\alpha \mathbf{F}+(1-\alpha) \mathbf{S}-\mathbf{\Sigma}\|^{2},
$$

which gives rise to the risk function

$$
\begin{aligned}
\mathrm{R}(\alpha) & =\mathrm{E}(\mathrm{L}(\alpha))=\sum_{\mathrm{i}=1}^{N} \sum_{\mathrm{j}=1}^{N} \mathrm{E}\left(\alpha \mathrm{f}_{\mathrm{ij}}+(1-\alpha) \mathrm{s}_{\mathrm{ij}}-\sigma_{\mathrm{ij}}\right)^{2} \\
& =\sum_{i=1}^{N} \sum_{j=1}^{N} \operatorname{Var}\left(\alpha \mathrm{f}_{\mathrm{ij}}+(1-\alpha) \mathrm{s}_{\mathrm{ij}}\right)+\left[\mathrm{E}\left(\alpha \mathrm{f}_{\mathrm{ij}}+(1-\alpha) \mathrm{s}_{\mathrm{ij}}-\sigma_{\mathrm{ij}}\right)\right]^{2} \\
& =\sum_{i=1}^{N} \sum_{j=1}^{N} \alpha^{2} \operatorname{Var}\left(\mathrm{f}_{\mathrm{ij}}\right)+(1-\alpha)^{2} \operatorname{Var}\left(\mathrm{s}_{\mathrm{ij}}\right)+2 \alpha(1-\alpha) \operatorname{Cov}\left(\mathrm{f}_{\mathrm{ij}}, \mathrm{s}_{\mathrm{ij}}\right)+\alpha^{2}\left(\phi_{\mathrm{ij}}-\sigma_{\mathrm{ij}}\right)^{2}
\end{aligned}
$$

The goal now is to minimize the risk $\mathrm{R}(\alpha)$ with respect to $\alpha$. Calculating the first two derivatives of $\mathrm{R}(\alpha)$ yields after some basic algebra

$$
\begin{aligned}
\mathrm{R}^{\prime}(\alpha) & =2 \sum_{i=1}^{N} \sum_{j=1}^{N} \alpha \operatorname{Var}\left(\mathrm{f}_{\mathrm{ij}}\right)-(1-\alpha) \operatorname{Var}\left(\mathrm{s}_{\mathrm{ij}}\right)+(1-2 \alpha) \operatorname{Cov}\left(\mathrm{f}_{\mathrm{ij}}, \mathrm{s}_{\mathrm{ij}}\right)+\alpha\left(\phi_{\mathrm{ij}}-\sigma_{\mathrm{ij}}\right)^{2} \\
\mathrm{R}^{\prime \prime}(\alpha) & =2 \sum_{i=1}^{N} \sum_{j=1}^{N} \operatorname{Var}\left(\mathrm{f}_{\mathrm{ij}}-\mathrm{s}_{\mathrm{ij}}\right)+\left(\phi_{\mathrm{ij}}-\sigma_{\mathrm{ij}}\right)^{2} .
\end{aligned}
$$

Setting $\mathrm{R}^{\prime}(\alpha)=0$ and solving for $\alpha^{*}$ we get

$$
\alpha^{*}=\frac{\sum_{i=1}^{N} \sum_{j=1}^{N} \operatorname{Var}\left(\mathrm{s}_{\mathrm{ij}}\right)-\operatorname{Cov}\left(\mathrm{f}_{\mathrm{ij}}, \mathrm{s}_{\mathrm{ij}}\right)}{\sum_{i=1}^{N} \sum_{j=1}^{N} \operatorname{Var}\left(\mathrm{f}_{\mathrm{ij}}-\mathrm{s}_{\mathrm{ij}}\right)+\left(\phi_{\mathrm{ij}}-\sigma_{\mathrm{ij}}\right)^{2}} .
$$

Since $\mathrm{R}(\alpha)^{\prime \prime}$ is positive everywhere, this solution is verified as a minimum of our risk function. It is easy to see that $\alpha^{*}=O(1 / T)$. Indeed, the following Theorem shows the first order asymptotic behavior of the optimal shrinkage intensity $\alpha^{*}$. 
Theorem 1 Let $\pi$ denote the sum of asymptotic variances of the entries of the sample covariance matrix scaled by $\sqrt{T}: \pi=\sum_{i=1}^{N} \sum_{j=1}^{N}$ AsyVar $\left[\sqrt{T} \mathrm{~s}_{\mathrm{ij}}\right]$. Similarly, let $\rho$ denote the sum of asymptotic covariances of the entries of the single-index covariance matrix with the entries of the sample covariance matrix scaled by $\sqrt{T}: \rho=\sum_{i=1}^{N} \sum_{j=1}^{N}$ AsyCov $\left[\sqrt{\mathrm{T}} \mathrm{f}_{\mathrm{ij}}, \sqrt{\mathrm{T}} \mathrm{s}_{\mathrm{ij}}\right]$. Finally, let $\gamma$ measure the misspecification of the single-index model: $\gamma=\sum_{i=1}^{N} \sum_{j=1}^{N}\left(\phi_{i j}-\sigma_{i j}\right)^{2}$. Then the optimal shrinkage $\alpha^{*}$ satisfies:

$$
\alpha^{*}=\frac{1}{T} \frac{\pi-\rho}{\gamma}+O\left(\frac{1}{T^{2}}\right)
$$

Proof of Theorem 1 Relation (3) implies that

$$
T \alpha^{*}=\frac{\sum_{i=1}^{N} \sum_{j=1}^{N} \operatorname{Var}\left(\sqrt{\mathrm{T}} \mathrm{s}_{\mathrm{ij}}\right)-\operatorname{Cov}\left(\sqrt{\mathrm{T}} \mathrm{f}_{\mathrm{ij}}, \sqrt{\mathrm{T}} \mathrm{s}_{\mathrm{ij}}\right)}{\sum_{i=1}^{N} \sum_{j=1}^{N} \operatorname{Var}\left(\mathrm{f}_{\mathrm{ij}}-\mathrm{s}_{\mathrm{ij}}\right)+\left(\phi_{\mathrm{ij}}-\sigma_{\mathrm{ij}}\right)^{2}} .
$$

By standard arguments, using the assumptions of iid data and finite fourth moments, it follows that

$\sum_{i=1}^{N} \sum_{j=1}^{N} \operatorname{Var}\left(\sqrt{\mathrm{T}} \mathrm{s}_{\mathrm{ij}}\right) \rightarrow \pi \quad \sum_{\mathrm{i}=1}^{\mathrm{N}} \sum_{\mathrm{j}=1}^{\mathrm{N}} \operatorname{Cov}\left(\sqrt{\mathrm{T}} \mathrm{f}_{\mathrm{ij}}, \sqrt{\mathrm{T}} \mathrm{s}_{\mathrm{ij}}\right) \rightarrow \rho \quad$ and $\quad \sum_{\mathrm{i}=1}^{\mathrm{N}} \sum_{\mathrm{j}=1}^{\mathrm{N}} \operatorname{Var}\left(\mathrm{f}_{\mathrm{ij}}-\mathrm{s}_{\mathrm{ij}}\right)=O\left(\frac{1}{\mathrm{~T}}\right)$.

Indeed, to show the first convergence, it is sufficient to focus on an arbitrary element $\pi_{i j}=$ AsyVar $\left[\sqrt{T} s_{\mathrm{ij}}\right]$. Without loss of generality assume that $E\left(x_{i t}\right)=E\left(x_{j t}\right)=0$. We start with the statistic

$$
\hat{\sigma}_{i j}=\frac{1}{T} \sum_{t=1}^{T} x_{i t} x_{j t}
$$

Note that $x_{i 1} x_{j 1}, \ldots, x_{i T} x_{j T}$ is an iid sequence with mean $\pi_{i j}$ and finite variance $\varrho_{i j}$, say. The standard CLT therefore implies that

$$
\sqrt{T}\left(\hat{\sigma}_{i j}-\sigma_{i j}\right) \Longrightarrow N\left(0, \varrho_{i j}^{2}\right)
$$

where $\Longrightarrow$ denotes convergence in distribution. Let $x_{i}=T^{-1} \sum_{t=1}^{T} x_{i t}$ and $x_{j}=T^{-1} \sum_{t=1}^{T} x_{j t}$. Then,

$$
\begin{aligned}
\sqrt{T}\left(\hat{\sigma}_{i j}-s_{i j}\right) & =\sqrt{T} \bar{x}_{i} \cdot \frac{1}{T} \sum_{t=1}^{T}\left(x_{j t}-x_{j}\right)+\sqrt{T} x_{j} \cdot \frac{1}{T} \sum_{t=1}^{T}\left(x_{i t}-x_{i}\right)+\sqrt{T} x_{i} \cdot x_{j} . \\
& =\sqrt{T} x_{i} \cdot x_{j} .
\end{aligned}
$$

By the standard CLT again, $\sqrt{T} x_{i}$. has a limiting normal distribution and is thus $O_{P}(1)$. On the other hand, $x_{j}$. converges to zero almost surely and is thus $o_{P}(1)$. Hence, it is easily seen that $\sqrt{T}\left(\hat{\sigma}_{i j}-s_{i j}\right)=o_{P}(1)$. By the convergence in distribution (7) and Slutzky's theorem, it follows that

$$
\sqrt{T}\left(s_{i j}-\sigma_{i j}\right) \Longrightarrow N\left(0, \varrho_{i j}\right) \text {. }
$$

Moreover, this implies that $\varrho_{i j}=\pi_{i j}$ and that $\operatorname{Var}\left(\sqrt{\mathrm{T}} \mathrm{s}_{\mathrm{ij}}\right) \rightarrow \pi_{\mathrm{ij}}$. Since we focused on an arbitrary element $\pi_{i j}$, the same argument can be used for the other elements as well. Combining 


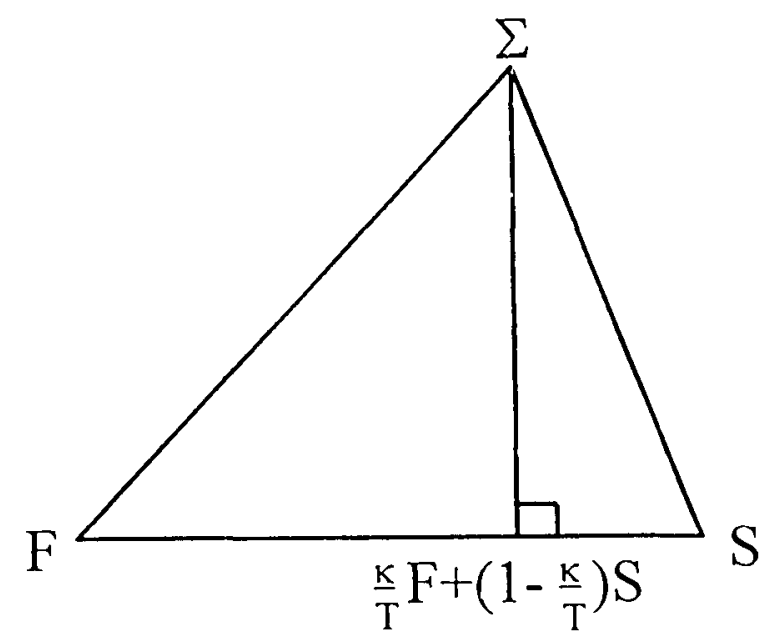

Figure 1: Geometric Interpretation of Theorem 1. The notion of orthogonality among $N$-dimensional symmetric matrices is defined by the inner product associated with the Frobenius norm.

the individual convergences (noting that there are a finite and fixed number of them), we arrive at $\sum_{i=1}^{N} \sum_{j=1}^{N} \operatorname{Var}\left(\sqrt{\mathrm{T}} \mathrm{s}_{\mathrm{ij}}\right) \rightarrow \pi$.

The convergence $\sum_{i=1}^{N} \sum_{j=1}^{N} \operatorname{Cov}\left(\sqrt{\mathrm{T}} \mathrm{f}_{\mathrm{ij}}, \sqrt{\mathrm{T}}_{\mathrm{ij}}\right) \rightarrow \rho$ is proved analogously.

Finally, a similar argument can be used to demonstrate that $\sum_{i=1}^{N} \sum_{j=1}^{N} \operatorname{Var}\left(\sqrt{\mathrm{T}}\left(\mathrm{f}_{\mathrm{ij}}-\mathrm{s}_{\mathrm{ij}}\right)\right)$ converges to a positive limit, which implies that $\sum_{i=1}^{N} \sum_{j=1}^{N} \operatorname{Var}\left(\mathrm{f}_{\mathrm{ij}}-\mathrm{s}_{\mathrm{ij}}\right)=\mathrm{O}(1 / \mathrm{T})$.

We thus have verified the claim (6). But (5) and (6) together imply (4) and this completes the proof.

The theorem shows that within the class of shrinkage intensities $\{\alpha=$ constant/T $\}$, the asymptotically optimal choice is given by constant $=\kappa$ with $\kappa=(\pi-\rho) / \gamma$. The analysis of the optimal constant $\kappa$ indicates the following. The weight placed on the single-index model increases in the error on the sample covariance matrix (through $\pi$ ) and decreases in the misspecification of the single-index model (through $\gamma$ ). An alternative interpretation of this solution is a geometric one: $\frac{\kappa}{T} \mathbf{F}+\left(1-\frac{\kappa}{T}\right) \mathbf{S}$ is (asymptotically) the orthogonal projection of the true covariance matrix $\boldsymbol{\Sigma}$ onto the line joining single-index model and sample covariance matrices (see Figure 1).

Note also the appearance of the term $\rho$ that measures the covariance between the estimation errors of $\mathbf{S}$ and $\mathbf{F}$ and was not present in the work of Frost and Savarino (1986). It is easier to understand within the empirical Bayesian interpretation of our shrinkage estimator: we can say that we have a prior based on the single-index model which we combine with sample information. Estimating the prior $\mathbf{F}$ from the same data set as $\mathbf{S}$ violates the pure Bayesian principle that prior and sample information should be independent. This is typical of the 
empirical Bayesian approach. Yet this violation is often ignored, and there is an "art" to choosing the prior so that the violation is not too damaging. We get rid of the need for such artistry by explicitly taking into account the correlation between prior and sample information through $\rho$.

Finally, the reader can verify that Theorem 1 is of general nature: nowhere did we use the fact that $\mathbf{F}$ is a single-index model estimator. Equation (4) stays the same as long as $\mathbf{F}$ is an asymptotically biased estimator of the covariance matrix and satisfies a set of weak regularity conditions.

\subsection{A Consistent Estimator of the Optimal Shrinkage Constant}

Note that $\frac{\kappa}{T} \mathbf{F}+\left(1-\frac{\kappa}{T}\right) \mathbf{S}$ is not a bona fide estimator because $\kappa$ depends on unobservables. Therefore, we need to find a consistent estimator for $\kappa=(\pi-\rho) / \gamma$. We can decompose $\pi$ into $\pi=\sum_{i=1}^{N} \sum_{j=1}^{N} \pi_{i j}$ where $\pi_{i j}=\operatorname{Asy} \operatorname{Var}\left[\sqrt{\mathrm{T}} \mathrm{s}_{\mathrm{ij}}\right], \rho$ into $\rho=\sum_{i=1}^{N} \sum_{j=1}^{N} \rho_{i j}$ where $\rho_{i j}=$ AsyCov $\left[\sqrt{\mathrm{T}} \mathrm{f}_{\mathrm{ij}}, \sqrt{\mathrm{T}} \mathrm{s}_{\mathrm{ij}}\right]$, and $\gamma$ into $\gamma=\sum_{i=1}^{N} \sum_{j=1}^{N} \gamma_{i j}$ where $\rho_{i j}=\left(\phi_{i j}-\sigma_{i j}\right)^{2}$. Standard asymptotic theory provides consistent estimators for $\pi_{i j}, \rho_{i j}$ and $\gamma_{i j}$.

Lemma $1 A$ consistent estimator for $\pi_{i j}$ is given by:

$$
p_{i j}=\frac{1}{T} \sum_{t=1}^{T}\left\{\left(x_{i t}-m_{i}\right)\left(x_{j t}-m_{j}\right)-s_{i j}\right\}^{2} .
$$

Proof of Lemma $1 p_{i j}$ is the usual estimator for the asymptotic variance of $s_{i j}$. It converges in probability to $\operatorname{Var}\left[\left(\mathrm{x}_{\mathrm{i} 1}-\mu_{\mathrm{i}}\right)\left(\mathrm{x}_{\mathrm{j} 1}-\mu_{\mathrm{j}}\right)\right]$, which is equal to $\pi_{i j}$.

Let $m_{0}$ denote the sample mean of market returns and $s_{i 0}$ the sample covariance of stock $i$ 's returns with the market.

Lemma 2 On the diagonal a consistent estimator of $\rho_{i i}$ is given by $r_{i i}=p_{i i}$, and for $i \neq j a$ consistent estimator of $\rho_{i j}$ is given by $r_{i j}=\frac{1}{T} \sum_{t=1}^{T} r_{i j t}$ where:

$$
\begin{aligned}
r_{i j t}= & \frac{s_{j 0} s_{00}\left(x_{i t}-m_{i}\right)+s_{i 0} s_{00}\left(x_{j t}-m_{j}\right)-s_{i 0} s_{j 0}\left(x_{0 t}-m_{0}\right)}{s_{00}^{2}}\left(x_{0 t}-m_{0}\right)\left(x_{i t}-m_{i}\right)\left(x_{j t}-m_{j}\right) \\
& -f_{i j} s_{i j} .
\end{aligned}
$$

Proof of Lemma 2 On the diagonal $f_{i i}=s_{i i}$, therefore $\rho_{i i}=\pi_{i i}$ can be consistently estimated by $r_{i i}=p_{i i}$. When $i \neq j$ we have: $f_{i j}=b_{i} b_{j} s_{00}=s_{i 0} s_{j 0} / s_{00}$, therefore the delta method yields:

$$
\begin{aligned}
\rho_{i j}= & \operatorname{AsyCov}\left[\sqrt{\mathrm{T}} \frac{\mathrm{s}_{\mathrm{i} 0} \mathrm{~s}_{\mathrm{j} 0}}{\mathrm{~s}_{00}}, \sqrt{\mathrm{T}} \mathrm{s}_{\mathrm{ij}}\right] \\
= & \frac{s_{j 0}}{s_{00}} \operatorname{AsyCov}\left[\sqrt{\mathrm{T}} \mathrm{s}_{\mathrm{i} 0}, \sqrt{\mathrm{T}} \mathrm{s}_{\mathrm{ij}}\right]+\frac{\mathrm{s}_{\mathrm{i} 0}}{\mathrm{~s}_{00}} \operatorname{AsyCov}\left[\sqrt{\mathrm{T}} \mathrm{s}_{\mathrm{j} 0}, \sqrt{\mathrm{T}} \mathrm{s}_{\mathrm{ij}}\right] \\
& -\frac{s_{i 0} s_{j 0}}{s_{00}^{2}} \operatorname{AsyCov}\left[\sqrt{\mathrm{T}} \mathrm{s}_{00}, \sqrt{\mathrm{T}} \mathrm{s}_{\mathrm{ij}}\right] .
\end{aligned}
$$


The usual estimator for AsyCov $\left[\sqrt{\mathrm{T}}_{\mathrm{k} 0}, \sqrt{\mathrm{T}} \mathrm{s}_{\mathrm{ij}}\right]$ where $k=0, k=i$ or $k=j$ is:

$$
\frac{1}{T} \sum_{t=1}^{T}\left\{\left(x_{k t}-m_{k}\right)\left(x_{0 t}-m_{0}\right)-s_{k 0}\right\}\left\{\left(x_{i t}-m_{i}\right)\left(x_{j t}-m_{j}\right)-s_{i j}\right\} .
$$

Plugging this estimator into Equation (9) and rearranging yields Equation (8).

Lemma 3 A consistent estimator for $\gamma_{i j}=\left(\phi_{i j}-\sigma_{i j}\right)^{2}$ is its sample counterpart $c_{i j}=\left(f_{i j}-\right.$ $\left.s_{i j}\right)^{2}$.

Proof of Lemma 3 This is because $f_{i j}$ and $s_{i j}$ are consistent estimators for $\phi_{i j}$ and $\sigma_{i j}$, respectively.

Now it is easy to construct an estimator for the optimal shrinkage constant.

Theorem $2 k=(p-r) / c$ is a consistent estimator for the optimal shrinkage constant $\kappa=$ $(\pi-\rho) / \gamma$.

Proof of Theorem 2 Under Assumption $4(\gamma>0)$, combining the results of Lemmata 1-3 proves the theorem.

Using this notation, the shrinkage estimator for the covariance matrix of stock returns that we recommend is:

$$
\widehat{\mathbf{S}}=\frac{k}{T} \mathbf{F}+\left(1-\frac{k}{T}\right) \mathbf{S}
$$

Different shrinkage targets would lend themselves just as well to a corresponding estimation of $\pi, \rho$ and $\gamma$. The formula for $r$ would need to be readjusted on a case-by-case basis, but the formulas for $p$ and $c$ would not change.

\section{Empirical Results}

We present empirical evidence on the performance of the shrinkage estimator defined in the last section. We compare it to existing estimators in terms of its ability to select portfolios of stocks with low out-of-sample variance.

\subsection{Portfolio Selection}

Consider a universe of $N$ stocks whose returns are distributed with mean vector $\boldsymbol{\mu}$ and covariance matrix $\boldsymbol{\Sigma}$. Markowitz (1952) defines the problem of portfolio selection as:

$$
\begin{aligned}
& \min _{\mathbf{w}} \mathbf{w}^{\prime} \Sigma \mathbf{w} \\
& \text { subject to } \mid \begin{array}{l}
\mathbf{w}^{\prime} 1=1 \\
\mathbf{w}^{\prime} \boldsymbol{\mu}=q
\end{array}
\end{aligned}
$$


where 1 denotes a conformable vector of ones and $q$ is the expected rate of return that is required on the portfolio. The well-known solution is:

$$
\begin{aligned}
\mathbf{w} & =\frac{C-q B}{A C-B^{2}} \boldsymbol{\Sigma}^{-1} \mathbf{1}+\frac{q A-B}{A C-B^{2}} \boldsymbol{\Sigma}^{-1} \boldsymbol{\mu} \\
\text { where } A & =\mathbf{1}^{\prime} \boldsymbol{\Sigma}^{-1} \mathbf{1}, \quad B=\mathbf{1}^{\prime} \boldsymbol{\Sigma}^{-1} \boldsymbol{\mu} \text { and } C=\boldsymbol{\mu}^{\prime} \boldsymbol{\Sigma}^{-1} \boldsymbol{\mu}
\end{aligned}
$$

Equation (12) shows that optimal portfolio weights depend on the inverse of the covariance matrix. This sometimes causes difficulty if the covariance matrix estimator is not invertible or if it is numerically ill-conditioned, which means that inverting it amplifies estimation error tremendously (Michaud, 1989). The shrinkage estimator $\widehat{\mathbf{S}}$ is the weighted average of two positive semi-definite matrices, one of which (F) is invertible, therefore it is invertible. Also, it inherits the good-conditioning of the single-index model estimator, not the ill-conditioning of the sample covariance matrix.

In practice, the covariance matrix is estimated from historical data available up to a given date, optimal portfolio weights are computed from this estimate, then the portfolio is formed on that date and held until the next rebalancing occurs. The performance of a covariance matrix estimator is measured by the variance of this optimal portfolio after it is formed. It is a measure of out-of-sample performance, or of predictive ability. An estimator that overfits in-sample data can turn out to work very poorly for portfolio selection, which is why imposing some structure is beneficial.

The other input into portfolio selection is the vector of expected returns. It is sometimes argued that estimating the covariance matrix well is less important than estimating the expected returns well. We believe that this view is profoundly misguided. First, the essence of mean-variance analysis is that there is a trade-off between risk and return, therefore any reduction in risk translates into an increase in expected returns. Second, having a good estimator of the covariance matrix helps us estimate more precisely the excess return associated with, for example, beta, size, or book-to-market by constructing portfolios that load on these characteristics and have low variance (this is related to the efficiency gain in running GLS cross-sectional regressions of stock returns on these characteristics). Third, it is not the role of just the statistician to determine expected returns, it is also the role of the economist, and of the portfolio manager who is paid to generate valuable private information about future stock price movements; whereas only statistics can generate information about the covariance matrix. This justifies why it is perfectly legitimate to concentrate on the covariance matrix alone without worrying about expected returns, as we do here.

\subsection{Data}

Stock returns were extracted from the Center for Research in Security Prices (CRSP) monthly database. The same procedure is repeated for every year from $t=1972$ to $t=1994$. We use data from August of year $t-10$ to July of year $t$ to estimate the covariance matrix of stock 
returns. ${ }^{3}$ Then on the first trading day in August of year $t$ we build a portfolio with minimum variance (according to this covariance matrix estimate) under certain constraints. We hold this portfolio until the last trading day in July of year $t+1$, at which time we liquidate it and start the process all over again. Thus, the in-sample period goes from August of year $t-10$ to July of year $t$, and the out-of-sample period goes from August of year $t$ to July of year $t+1$. The main quantity of interest is the out-of-sample standard deviation of this investment strategy over the 23-year period from August 1972 to July 1995. This is a predictive test, in the sense that our investment strategy does not require any hindsight.

In August of year $t$, we consider the universe of common stocks traded on the New York Stock Exchange (NYSE) and the American Stock Exchange (AMEX) with valid CRSP returns for the last 120 months and valid Standard Industrial Classification (SIC) codes. The resulting number of stocks varies across years between $N=909$ and $N=1,314$.

We consider two minimum variance portfolios: the global minimum variance portfolio, and the portfolio with minimum variance under the constraint of having $20 \%$ expected return. In both cases short sales are allowed, and no additional restriction is imposed (except that weights sum up to one). It is common practice in the investment community to impose client-specific constraints on portfolio weights or to minimize active risk with respect to an exogenously specified benchmark, but we abstract from that in order to have a "maximum stress" test of the performance of the covariance matrix estimator. For expected returns, we just take the average realized return over the last 10 years. This may or may not be a good predictor of future expected returns, but our goal is not to predict expected returns: it is only to show what kind of reduction in out-of-sample variance our method yields under a fairly reasonable linear constraint.

\subsection{Competing Estimators}

Apart from our shrinkage estimator, we consider the following covariance matrix estimators proposed in the literature.

Identity The simplest model is to assume that the covariance matrix is a scalar multiple of the identity matrix. This is the assumption implicit in running an Ordinary Least Squares (OLS) cross-sectional regression of stock returns on stock characteristics, as Fama and MacBeth (1973) and their successors do. Interestingly, it yields the same weights for the minimum variance portfolios as a two-parameter model where all variances are equal to one another and all covariances are equal to one another. This two-parameter model is discussed by Jobson and Korkie (1980) and by Frost and Savarino (1986).

\footnotetext{
${ }^{3}$ We rebalance every August because the earliest AMEX stock returns available from CRSP are in August 1962.
} 
Constant Correlation Elton and Gruber (1973) recommend a model where every pair of stocks has the same correlation coefficient. Thus, there are $N+1$ parameters to estimate: the $N$ individual variances, and the constant correlation coefficient.

Pseudo-Inverse It is impossible to use the sample covariance matrix directly for portfolio selection when the number of stocks $N$ exceeds the number of historical returns $T$, which is the case here. The problem is that we need the inverse of the sample covariance matrix, and it does not exist. One possible trick to get around this problem is to use the pseudo-inverse, also called generalized inverse or Moore-Penrose inverse. Replacing the inverse of the sample covariance matrix by the pseudo-inverse into Equation (12) yields well-defined portfolio weights.

Market Model This is the single-index covariance matrix of Sharpe (1963), which is defined in Section 2.3.

Industry Factors This refinement of the single-index model assumes that market residuals are generated by industry factors:

$$
x_{i t}=\alpha_{i}+\beta_{i} x_{0 t}+\sum_{k=1}^{K} c_{i k} z_{k t}+\varepsilon_{i t}
$$

where $K$ is the number of industry factors, $c_{i k}$ is a dummy variable equal to one if stock $i$ belongs to industry category $k, z_{k t}$ is the return to the $k$-th industry factor in period $t$, and $\varepsilon_{k t}$ denotes residuals that are uncorrelated to the market, to industry factors, and to each other. Every stock is assigned to one of the 48 industries defined by Fama and French (1997). This high number of factors is similar to the one used by the company BARRA to produce commercial multi-factor estimates of the covariance matrix (Kahn, 1993). ${ }^{4}$ Industry factor returns are defined as the return to an equally-weighted portfolio of the stocks from this industry in our sample.

Principal Components An alternative approach to multi-factor models is to extract the factors from the sample covariance matrix itself using a statistical method such as principal components. Some investment consultants such as Advanced Portfolio Technologies successfully use a refined version of this approach (Bender and Blin, 1997). Since principal components are chosen solely for their ability to explain risk, fewer factors are necessary, but they do not have any direct economic interpretation. ${ }^{5}$ A sophisticated test by Connor and Korajczyk (1993) finds between four and seven factors for the NYSE and AMEX over 1967-1991, which is in the same range as the original test by Roll and Ross (1980). The number of factors that we use here is five.

\footnotetext{
${ }^{4}$ In addition, BARRA use proprietary methods, including other factors that are not industry-based, therefore this is not a test of their performance.

${ }^{5}$ Except for the first factor, which is highly correlated with the market index.
} 
Shrinkage Towards Identity A related shrinkage estimator of Ledoit and Wolf (2000) uses a scalar multiple of the identity matrix as shrinkage target; note that their estimator, under a different asymptotic framework, is suggested for general situations where no "natural" shrinking target exists. This seems suboptimal for stock returns, since stock returns have different variances and mainly positive covariances. Hence, it seems beneficial to use a shrinkage target which incorporates this knowledge, such as the single-index covariance matrix. Nevertheless, we include this estimator.

Shrinkage Towards Market This is the estimator $\widehat{\mathbf{S}}$ defined in (10).

\subsection{Out-of-Sample Standard Deviations}

For every one of the eight estimators described in the previous subsection, we compute the out-of-sample (annualized) standard deviation of the minimum variance portfolios as per Section 3.2. The results are in Table 1.

\begin{tabular}{|l||cc|cc|}
\hline & \multicolumn{2}{|c|}{$\begin{array}{c}\text { Std. Deviation } \\
\text { Unconstrained }\end{array}$} & \multicolumn{2}{|c|}{$\begin{array}{c}\text { Std. Deviation } \\
\text { Constrained }\end{array}$} \\
\hline \hline Identity & 17.75 & $(0.44)$ & 17.94 & $(0.42)$ \\
\hline Constant Correlation & 14.27 & $(0.19)$ & 16.30 & $(0.29)$ \\
\hline Pseudo-Inverse & 12.37 & $(0.23)$ & 13.73 & $(0.32)$ \\
\hline Market Model & 12.00 & $(0.16)$ & 13.77 & $(0.27)$ \\
\hline Industry Factors & 10.84 & $(0.17)$ & 12.32 & $(0.23)$ \\
\hline Principal Components & 10.31 & $(0.16)$ & 11.30 & $(0.22)$ \\
\hline Shrinkage To Identity & 10.21 & $(0.17)$ & 11.11 & $(0.21)$ \\
\hline Shrinkage To Market & 9.55 & $(0.15)$ & 10.43 & $(0.20)$ \\
\hline
\end{tabular}

Table 1: Risk of Minimum Variance Portfolios. "Unconstrained" refers to the global minimum variance portfolio, while "constrained" refers to the minimum variance portfolio with $20 \%$ expected return. Standard deviation is measured out-of-sample at the monthly frequency, annualized through multiplication by $\sqrt{12}$, and expressed in percents. Standard errors on these standard deviation estimates are reported in parenthesis.

We can see that naive diversification (one dollar in every stock) performs the worst, while the shrinkage estimator developed above performs the best; somewhat surprisingly, maybe, shrinking towards the identity is second best and also beats all of the previously suggested methods. The $t$-statistics of whether $\widehat{\mathbf{S}}$ yields portfolios with lower variance than its seven competitors range from 2.73 (against the other shrinkage estimator) to 7.39 (against the constant correlation model).

To assess economic significance, the rule of thumb is that a decrease of two basis points in standard deviation corresponds to an increase of one basis points in expected returns, 
using standard numbers for the risk-return tradeoff. ${ }^{6}$ For example, gains over the two multifactor models are 43 and 94 basis points respectively in terms of average returns for the constrained portfolio. By this metric, improvement over the two multi-factor models and the shrinkage towards the identity is reasonable. Improvement over the other estimators, including the sample covariance matrix and the single-index model (the ones which we are combining together), is large.

\subsection{Weight Distribution}

We also report descriptors of the weights of the global minimum variance portfolio: turnover, short interest, lowest and highest weight. When a stock has missing out-of-sample observations we assume that it earns the riskfree rate. Thus, it is important to report the cash position, defined as the total amount invested in stocks with missing observations. These weight descriptors are in Table 2.

\begin{tabular}{|l||r|r|r|r|r|}
\hline & Turnover & $\begin{array}{c}\text { Short } \\
\text { Interest }\end{array}$ & $\begin{array}{c}\text { Lowest } \\
\text { Weight }\end{array}$ & $\begin{array}{c}\text { Highest } \\
\text { Weight }\end{array}$ & $\begin{array}{c}\text { Cash } \\
\text { Position }\end{array}$ \\
\hline \hline Identity & 6 & 0 & 0.09 & 0.09 & 2.50 \\
\hline Constant Correlation & 24 & 68 & -0.17 & 2.86 & -1.40 \\
\hline Pseudo-Inverse & 96 & 99 & -1.16 & 1.12 & 1.46 \\
\hline Market Model & 23 & 51 & -0.41 & 2.10 & 0.13 \\
\hline Industry Factors & 43 & 89 & -1.08 & 2.81 & 0.54 \\
\hline Principal Components & 49 & 80 & -0.84 & 2.95 & 0.78 \\
\hline Shrinkage To Identity & 71 & 113 & -1.23 & 1.19 & 0.99 \\
\hline Shrinkage To Market & 61 & 98 & -1.01 & 3.81 & 0.78 \\
\hline
\end{tabular}

Table 2: Weight Descriptors. These are expressed in percents and averaged over the 23 years in our sample. A short interest of $68 \%$ means that for every dollar invested in the portfolio we short 68 cents worth of stocks, while buying $\$ 1.68$ worth of other stocks. Annual turnover above $100 \%$ is possible because of short sales.

If we wanted to do portfolio selection in practice, all these minimum variance portfolios would have too much of a short interest to be really attractive. But absolutely no effort was made to control for this characteristic, therefore there is considerable room for improving it without substantially degrading the performance reported in Table 1. In our view, Table 2 indicates that the weight distribution generated by the shrinkage estimator is acceptable overall.

The cash position due to missing out-of-sample observations is always very small, therefore the standard deviations in Table 1 do indeed correspond to portfolios almost fully invested in equities. Otherwise, it would be easy to get low risk simply by holding cash.

\footnotetext{
${ }^{6}$ Specifically: $8.5 \%$ market risk premium and $17 \%$ market standard deviation.
} 


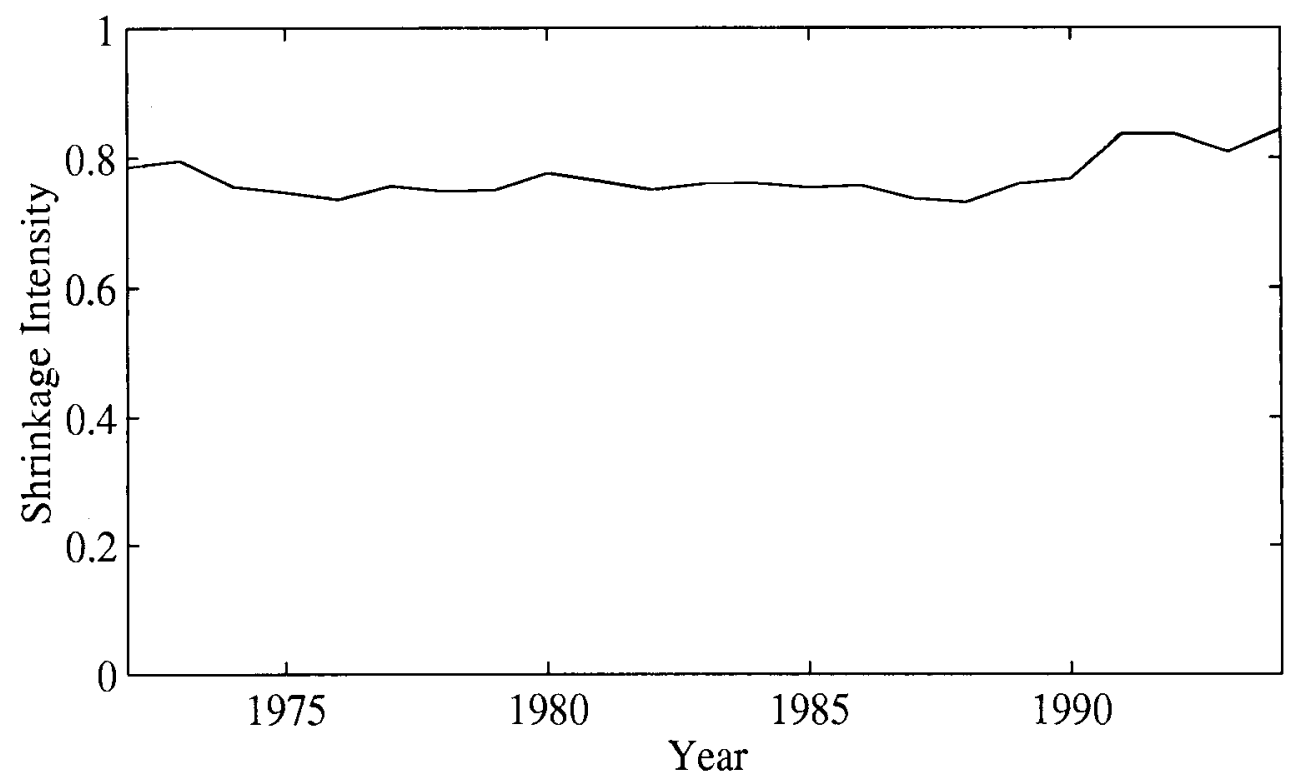

Figure 2: Optimal Shrinkage Intensity Estimate. This is the weight $k / T$ placed on the singleindex model covariance matrix, as defined by Theorem 2 .

\subsection{Shrinkage Intensity}

Figure 2 shows how the estimate of the optimal shrinkage intensity evolves through the 23 years in our sample.

It is always between zero and one, which is what we would expect. It is remarkably stable through time. In particular, this implies that there is very little estimation error, as predicted by Theorem 2. It is fairly high: around $80 \%$. This means that there is four times as much estimation error in the sample covariance matrix as there is bias in the single-index model. While $k / T$ is an asymptotically negligible correction, we see that in practice it can make a big difference.

In spite of the large number of stocks, the computations are remarkably fast. Using a desktop personal computer and a Matlab program, it took us about five minutes to compute the optimal shrinkage intensity, the covariance matrix estimator $\widehat{\mathbf{S}}$, and the minimum variance portfolio weights for $N=1,000$ stocks.

The term $r$ in the formula for the optimal shrinkage intensity, which is one of the major innovations of this paper, turns out to be critical in practice. If we had omitted it, all the shrinkage intensities would have been well above one and thus meaningless.

\section{Conclusion}

We have developed a flexible method for imposing some structure into a large-dimensional estimation problem, namely the problem of estimating the covariance matrix of a large number 
of stock returns. The crux of the method is to shrink the unbiased but very variable sample covariance matrix towards the biased but less variable single-index model covariance matrix and to thereby obtain a more efficient estimator. In addition, the resulting estimator is invertible and well-conditioned, which is of crucial importance in case one needs to estimate the inverse of the true covariance matrix.

The practical problem in applying our method is to to determine the shrinkage intensity, that is, the amount of shrinkage of the sample covariance matrix towards the single-index model covariance matrix. The problem was solved by first demonstrating that the optimal shrinking intensity, to second order, behaves like a constant over the sample size, and by then providing a way to consistently estimate that constant. In practice, one uses the estimated constant over the sample size as the shrinkage intensity.

As a by-product, this paper also reduces the dependence on multi-factor models, which are surrounded by unresolved questions about the number of factors and their identity. There has been much debate over whether factors should have an economic interpretation or should explain a lot of the variation in stock returns. Ideally, they should do both. By this (admittedly stringent) criterion, there is one obvious factor: the market. We are not saying that extramarket covariance is negligible, but that it lacks strong factor structure. This is precisely why we have developed a way to account for extra-market covariance without fitting it into an arbitrary factor structure.

We compared the performance of the shrinkage method to that of various previously suggested estimators for the covariance matrix of stock returns. Performance was measured in terms of out-of-sample standard deviation of minimum variance stock portfolios, where the estimated covariance matrix is the input of the well-known portfolio selection method of Markowitz (1952). Our method improved upon all the other estimators included in the study.

It should be pointed out that portfolio selection is only one of many problems that benefit from a more accurate estimation of the covariance matrix of stock returns. For example, consider tests of the Capital Asset Pricing Model (CAPM) that consist of predictive crosssectional regressions of average stock returns on betas and various stock attributes. Most studies use Ordinary Least Squares (OLS) regressions instead of Generalized Least Squares (GLS) regressions for lack of an invertible and accurate estimator of the covariance matrix of stock returns. This state of affairs is regrettable because GLS is superior to OLS for several reasons: it is more powerful; its economic interpretation is clearer because it is directly related to portfolio selection (e.g., see Kandel and Stambaugh, 1995); and correlation across stocks is a prominent feature of returns that should not be ignored. 


\section{References}

Bender, S. and Blin, J. (1997). Arbitrage and the structure of risk: A mathematical analysis. Modern Finance: 1-32.

Connor, G. and Korajczyk, R. A. (1992). The arbitrage pricing theory and multifactor models of asset returns. In Finance Handbook. R. Jarrow, V. Maksimovic and W. Ziemba, eds.

Connor, G. and Korajczyk, R. A. (1993). A test for the number of factors in an approximate factor model. Journal of Finance, 48:1263-1291.

Efron, B. and Morris, C. (1977). Stein's paradox in statistics. Scientific American, 237:119-127.

Elton, E. J. and Gruber, M. J. (1973). Estimating the dependence structure of share prices. Journal of Finance, 28:1203-1232.

Fama, E. F. and French, K. R. (1997). Industry costs of equity. Journal of Financial Economics, 43:153-193.

Fama, E. F. and MacBeth, J. (1973). Risk, return and equilibrium: Empirical tests. Journal of Political Economy, 81:607-636.

Frost, P. A. and Savarino, J. E. (1986). An empirical Bayes approach to portfolio selection. Journal of Financial and Quantitative Analysis, 21:293-305.

Jobson, J. D. and Korkie, B. (1980). Estimation for Markowitz efficient portfolios. Journal of the American Statistical Association, 75:544-554. Applications Section.

Kandel, S. and Stambaugh, R. F. (1995). Portfolio inefficiency and the cross-section of expected returns. Journal of Finance, 50:157-184.

Ledoit, O. and Wolf, M. (2000). A well-conditioned estimator for large dimensional covariance matrices. Working paper, Departamento de Estadística y Econometría, Universidad Carlos III de Madrid.

Markowitz, H. (1952). Portfolio selection. Journal of Finance, 7:77-91.

Michaud, R. O. (1989). The Markowitz optimization enigma: Is 'optimized' optimal? Financial Analysts Journal, 45:31-42.

Muirhead, R. J. (1987). Developments in eigenvalue estimation. Advances in Multivariate Statistical Analysis, 277-288.

Roll, R. (1977). A critique of the asset pricing theory's test; part I: On past and potential testability of the theory. Journal of Financial Economics, 4:129-176.

Roll, R. and Ross, S. A. (1980). An empirical investigation of the arbitrage pricing theory. Journal of Finance, 35:1073-1103. 
Ross, S. A. (1976). The arbitrage theory of capital asset pricing. Journal of Economic Theory, 13:341-360.

Sharpe, W. F. (1963). A simplified model for portfolio analysis. Management Science: 9:277293.

Stein, C. (1956). Inadmissibility of the usual estimator for the mean of a multivariate normal distribution. In Neyman, J., editor, Proceedings of the Third Berkeley Symposium on Mathematical and Statistical Probability, pages 197-206. University of California, Berkeley. Volume I. 